\title{
Principles of electric-dipole-allowed optical control of molecular chirality
}

\author{
Paul Brumer \\ Chemical Physics Theory Group, Department of Chemistry, University of Toronto, Toronto, Canada M5S $3 H 6$
}

Einat Frishman and Moshe Shapiro

Chemical Physics Department, The Weizmann Institute of Science, Rehovot, Israel 76100

(Received 12 July 2001; published 5 December 2001)

\begin{abstract}
Conditions for achieving "optical asymmetric synthesis," an example of controlled chiral symmetry breaking, using the electric-dipole light-field interaction are derived. These include scenarios in which neither the medium nor the light is chiral by itself. Specifically, parity requirements are used to show that any optical scenario in which the dynamics of the molecule depends on the overall sign of the electric field allows for control over the production of one chiral species in preference to its mirror image. A sample laser-molecule scenario is used to demonstrate these conditions.
\end{abstract}

DOI: 10.1103/PhysRevA.65.015401

PACS number(s): 32.80.Qk, 11.30.Rd

The ability to produce a specific broken-symmetry system, and in particular, a chiral system of specific handedness, in preference to its equal-energy broken-symmetry forms, is of great interest, both practically as well as theoretically. Examples of broken-symmetry systems of interest include a pair of asymmetric quantum wells, one being the mirror image of the other; two heteronuclear molecules aligned in a DC electric field; and a 1:1 mixture (called a "racemic" mixture) of chiral molecules and their mirror images (such pairs are called "enantiomers"). Although the results described below may be applied to a rather general class of brokensymmetry systems, we focus here on the conversion of a racemic mixture to a single enantiomeric form, a process we term "optical asymmetric synthesis." Our interest is in identifying general conditions under which linearly polarized light may be used to achieve this goal.

The use of circularly polarized light [1-3] to selectively enhance a desired enantiomer, results in a very small effect for most molecules. The reason for this may be traced to the reliance on the presence of the weak molecular magnetic dipole. Alternatively, we can explain the minuteness of the effect by noting that although circularly polarized light is chiral, this chirality, which is due to the combined sense of the rotation of the electric (or magnetic) field and the direction of propagation, is hardly felt by the molecule due to the differences of at least three orders of magnitudes between the wavelength of light in the visible range and the molecular size.

In contrast, coherent control methods that utilize the far stronger electric-dipole interaction, in conjunction with either polarization $\left(M_{J}\right.$ selection) of photofragments [4] or of the initial racemic mixture $[5,6]$, or orientation $[7,8]$ of the initial sample, have been shown theoretically to yield a very high degree of enantio selectivity. In the former cases [4,5], linearly polarized light was used, so that neither the system, nor the light, was chiral by itself. As discussed below, chiral molecules of specific handedness may be generated, in a process we term "optical asymmetric synthesis," by combining a phase-specific electromagnetic field with a polarized racemic mixture.

In this Brief Report we derive the general conditions under which an optical asymmetric synthesis based purely on the electric-dipole light-field interaction is possible. The conditions are completely general, taking into account the properties of both the medium and the incident light.

Consider a molecule, described by the total Hamiltonian (including electrons and nuclei) $H_{M}$. Within the BornOppenheimer approximation adopted below, the nuclear wave function associated with the ground electronic state has two enantiomers denoted $L$ and $D$, related to one another through inversion $\mathcal{I}$. Specifically, $H_{M}$ eigenstates describing $L$ and $D$ and are denoted $\left|L_{i}\right\rangle$ and $\left|D_{i}\right\rangle(i=1,2,3, \ldots$,$) and$ satisfy

$$
\mathcal{I}\left|L_{i}\right\rangle=-\left|D_{i}\right\rangle ; \quad \mathcal{I}\left|D_{i}\right\rangle=-\left|L_{i}\right\rangle
$$

The dipole interaction of this molecule with an incident timedependent electric field $\mathbf{E}(t)$ is described by the Hamiltonian

$$
H(\mathbf{E})=H_{M}-\mu \times \mathbf{E} .
$$

Here, $\mu$ is the total dipole operator, including both electron and nuclear contributions, and we have explicitly indicated the dependence of the Hamiltonian on the electric field. Consider now the effect of inversion on $H$. Since $\mathcal{I}$ operates on the coordinates of the molecule, we first note that it reverses the sign of the dipole operator, i.e, $\mathcal{I}^{\dagger} \mu \mathcal{I}=-\mu$. Second, we note that the molecular Hamiltonian is invariant to the action of $\mathcal{I}$. Thus, since $\mathcal{I}^{\dagger}=\mathcal{I}$, that $\left[H_{M}, \mathcal{I}\right]=0$. Combining the above results, we have [9] that $\mathcal{I} H(\mathbf{E}) \mathcal{I}=H(-\mathbf{E})$, where $H(-\mathbf{E})=H_{M}+\mu \times \mathbf{E}$. This implies, defining $U(\mathbf{E})$ and $U$ $(-\mathbf{E})$ as the propagators corresponding to dynamics under $H(\mathbf{E})$ and $H(-\mathbf{E})$, respectively, that

$$
U(\mathbf{E}) \mathcal{I}=\mathcal{I} U(-\mathbf{E}) .
$$

To expose the underlying principles allowing optical asymmetric synthesis, consider irradiating a racemic mixture of $D$ and $L$ in its ground electronic-state with an electric field $\mathbf{E}$ and examine the difference $\delta$ between the amount of $D$ and $L$ formed. We consider first the coherent process using transform limited light in the absence of collisions. Then, the difference $\delta$ is given by 


$$
\begin{aligned}
\delta= & \sum_{i} P_{i} \quad \sum_{j}\left[\left|\left\langle D_{j}|U(\mathbf{E})| D_{i}\right\rangle\right|^{2}+\left|\left\langle D_{j}|U(\mathbf{E})| L_{i}\right\rangle\right|^{2}\right] \\
& -\left[\left|\left\langle L_{j}|U(\mathbf{E})| D_{i}\right\rangle\right|^{2}+\left|\left\langle L_{j}|U(\mathbf{E})| L_{i}\right\rangle\right|^{2}\right]
\end{aligned}
$$

where $P_{i}$ is the probability of state $\left|L_{i}\right\rangle$ and $\left|D_{i}\right\rangle$ in the initial mixed state. (Since the initial state is a racemic mixture, the states $\left|L_{i}\right\rangle$ and $\left|D_{i}\right\rangle$ appear with equal probability.) If $\delta=0$, then there is no control over the chirality in the scenario defined by $U(\mathbf{E})$.

To determine the conditions under which $\delta$ is nonzero, we rewrite Eq. (4) as

$$
\begin{aligned}
\delta= & \sum_{i} P_{i} \quad \sum_{j}\left[\left|\left\langle D_{j}|U(\mathbf{E})| D_{i}\right\rangle\right|^{2}-\left|\left\langle L_{j}|U(\mathbf{E})| L_{i}\right\rangle\right|^{2}\right] \\
& +\left[\left|\left\langle D_{j}|U(\mathbf{E})| L_{i}\right\rangle\right|^{2}-\left|\left\langle L_{j}|U(\mathbf{E})| D_{i}\right\rangle\right|^{2}\right]
\end{aligned}
$$

and recast the second and third terms using

$$
\begin{aligned}
\left|\left\langle L_{j}|U(\mathbf{E})| L_{i}\right\rangle\right|^{2} & =\left|\left\langle D_{j}\left|\mathcal{I}^{\dagger} U(\mathbf{E}) \mathcal{I}\right| D_{i}\right\rangle\right|^{2} \\
& =\left|\left\langle D_{j}|U(-\mathbf{E})| D_{i}\right\rangle\right|^{2}, \\
\left|\left\langle D_{j}|U(\mathbf{E})| L_{i}\right\rangle\right|^{2} & =\left|\left\langle D_{j}|U(\mathbf{E}) \mathcal{I}| D_{i}\right\rangle\right|^{2} \\
& =\left|\left\langle D_{j}|\mathcal{I} U(-\mathbf{E})| D_{i}\right\rangle\right|^{2} \\
& =\left|\left\langle L_{j}|U(-\mathbf{E})| D_{i}\right\rangle\right|^{2},
\end{aligned}
$$

giving

$$
\begin{aligned}
\delta= & \sum_{i} P_{i} \quad \sum_{j}\left[\left|\left\langle D_{j}|U(\mathbf{E})| D_{i}\right\rangle\right|^{2}-\left|\left\langle D_{j}|U(-\mathbf{E})| D_{i}\right\rangle\right|^{2}\right] \\
& +\left[\left|\left\langle L_{j}|U(-\mathbf{E})| D_{i}\right\rangle\right|^{2}-\left|\left\langle L_{j}|U(\mathbf{E})| D_{i}\right\rangle\right|^{2}\right]
\end{aligned}
$$

Equation (7), the essential result of this report, provides the general condition under which electric fields, assuming a dipole interaction, can break the right-left symmetry of the initial state, and result in enhanced production of a desired enantiomer. Specifically, the difference between the amount of $D$ and $L$ formed is seen to depend entirely on the difference between the molecular dynamics when irradiated by $\mathbf{E}$ and by $\mathbf{- E}$. Hence, barring cancellation of matrix elements of different $j$, any scenario where the dynamics of the molecules depends on the overall sign of the electric field may give a nonzero enantiomeric excess and a breaking of the left-right symmetry, even for cases with initial achiral precursors. Note that the fact that molecular dynamics may depend on the phase of the incident electric field is well substantiated $[10,11]$, but its utility for asymmetric synthesis is only evident from this result. Finally, note that the result is completely consistent with symmetry-based arguments that can usefully provide conditions under which $\delta$ must equal zero. For example, a racemic mixture of thermally equilibrated molecules is rotationally invariant. Hence, any rotation that converts $\mathbf{E}$ to $-\mathbf{E}$ could not, in this case, result in enantiomeric control. In particular, in this case, the sum over $M_{J}$ (where $M_{J}$ is the component of the total angular momentum along the direction of laser polarization) implicit in the sum over $P_{i}$ in Eq. (7) would result in $\delta=0$. By contrast, for

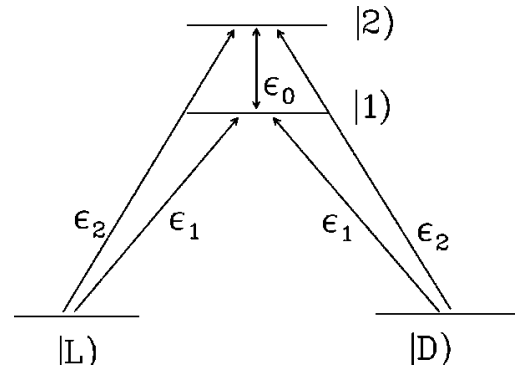

FIG. 1. Model system and laser scenario, as described in text.

example, a racemic mixture of $M_{J}$ polarized molecules irradiated with linearly polarized light [5] gives nonzero $\delta$. Other $\delta \neq 0$ examples emanating from Eq. (7) are also expected to display similar nontraditional characteristics.

Both qualitative and quantitative applications of Eq. (7) are possible. Qualitatively, for example, a traditional scheme where the ground electronic state of $L$ and $D$ are incoherently excited to bound levels of an excited state, gives $\delta=0$. This is because all processes connecting the initial and final $\left|L_{i}\right\rangle$ and $\left|D_{i}\right\rangle$ states, i.e., contributions to the matrix elements in Eq. (7), are even in the power of the electric field. Hence, propagation under $\mathbf{E}$ and $-\mathbf{E}$ are identical. By contrast, consider the four-level model scheme in Fig. 1, where the states $\left|D_{1}\right\rangle$ and $\left|L_{1}\right\rangle$ are denoted $|D\rangle$ and $|L\rangle$. (E.g., these may be four levels of fixed $M_{J}$ ). In this enhanced version of the scenario introduced in Ref. [5], two states $|1\rangle$ and $|2\rangle$ of energy $E_{1}$ and $E_{2}$, which are associated with an excited electronic state, are coupled to the ground state at energies $E_{D}$ $=E_{L}$ by two narrow pulses $\varepsilon_{1}(t), \varepsilon_{2}(t)$ of linearly polarized coherent light. Here the rovibrational component of $|1\rangle$ is symmetric with respect to $\mathcal{I}$, whereas $|2\rangle$ is antisymmetric [12]. The latter two levels are coupled to one another by an additional pulse of coherent linearly polarized light of amplitude $\varepsilon_{0}(t)$. When $\varepsilon_{0}(t) \neq 0$, there exist processes connecting the initial and final $|L\rangle$ and $|D\rangle$ states that are of the form $|L\rangle \rightarrow|1\rangle \rightarrow|2\rangle \rightarrow|D\rangle$, and hence, there are terms in Eq. (7) that are odd in the power of the electric field. One therefore anticipates the possibility of altering the enantiomeric excess using this combination of pulses, providing the basis for the control results reported in Ref. [5]. Further, if $\varepsilon_{0}=0$, then the situation reverts to the case discussed above, where only processes even in the electric field contribute to transitions between the initial $|D\rangle,|L\rangle$, and final $|D\rangle,|L\rangle$ transitions, and hence control over the enantiomeric excess is lost. For this reason, the $\varepsilon_{0}(t)$ coupling laser is crucial to enantiomeric control. This qualitative picture is substantiated quantitatively, below.

Quantitatively, the time evolution of the system shown in Fig. 1 is given by the wave function

$$
\begin{aligned}
|\Psi(t)\rangle= & b_{D}(t) \exp \left(-i E_{D} t / \hbar\right)|D\rangle+b_{L}(t) \exp \left(-i E_{L} t / \hbar\right)|L\rangle \\
& +b_{1}(t) \exp \left(-i E_{1} t / \hbar\right)|1\rangle \\
& +b_{2}(t) \exp \left(-i E_{2} t / \hbar\right)|2\rangle .
\end{aligned}
$$

Inserting Eq. (8) into the time-dependent Schrödinger equation, invoking the rotating wave approximation, and noting 
the symmetry properties of the nuclear component of $|1\rangle$ and $|2\rangle$ gives a set of equations [5] that may be solved numerically for the time dependence of the coefficients $b_{i}(t)$. Specifically,

$$
\begin{gathered}
\dot{b}_{1}=i \exp \left(i \Delta_{1} t\right) \Omega_{D, 1}^{*}\left[b_{D}+b_{L}\right]+i \exp \left(i \Delta_{0} t\right) \Omega_{0}^{*} b_{2}, \\
\dot{b}_{2}=i \exp \left(i \Delta_{2} t\right) \Omega_{D, 2}^{*}\left[b_{D}-b_{L}\right]+i \exp \left(-i \Delta_{0} t\right) \Omega_{0} b_{1}, \\
\dot{b}_{D}=i \exp \left(-i \Delta_{1} t\right) \Omega_{D, 1} b_{1}+i \exp \left(-i \Delta_{2} t\right) \Omega_{D, 2} b_{2}, \\
\dot{b}_{L}=i \exp \left(-i \Delta_{1} t\right) \Omega_{D, 1} b_{1}-i \exp \left(-i \Delta_{2} t\right) \Omega_{D, 2} b_{2},
\end{gathered}
$$

where the Rabi frequencies $\Omega_{i j}(t) \equiv \mu_{i j} \varepsilon_{j}(t) / \hbar, i$ $=D, L, \quad j=1,2, \Omega_{0} \equiv \mu_{21} \varepsilon_{0}(t) / \hbar$, and detunings $\Delta_{j} \equiv \omega_{j D}$ $-\omega_{j}, \Delta_{0} \equiv \omega_{21}-\omega_{0}$, where $\mu_{i j} \equiv\left\langle i\left|\mu \times \hat{\epsilon}_{k}\right| j\right\rangle$, with $i$ $=D, L ; k=0,1,2$, and $j=1,2$. Here, $\omega_{i j}=\left(E_{i}-E_{j}\right) / \hbar$ and $\hat{\epsilon}_{k}$ defines the direction of the linearly polarized pulse $\varepsilon_{k}(t)$. We take the pulses $\varepsilon_{1}$ and $\varepsilon_{2}$ much narrower in bandwidth than $\omega_{21}$, and neglect the effect of pulse 1 on level 2 and of pulse 2 on level 1.

Analytically, the solution to Eq. (9) depends on the sign of $\mathbf{E}$ when $\varepsilon_{0} \neq 0$, Specifically, changing $\mathbf{E}$ to $-\mathbf{E}$ means changing all $\varepsilon_{j}(t)$ to $-\varepsilon_{j}(t)$. Doing so, and defining $b_{1}^{\prime}=$ $-b_{1}$ and $b_{2}^{\prime}=-b_{2}$ converts Eq. (9) into

$$
\begin{gathered}
\dot{b}_{1}^{\prime}=i \exp \left(i \Delta_{1} t\right) \Omega_{D, 1}^{*}\left[b_{D}+b_{L}\right]-i \exp \left(i \Delta_{0} t\right) \Omega_{0}^{*} b_{2}^{\prime}, \\
\dot{b}_{2}^{\prime}=i \exp \left(i \Delta_{2} t\right) \Omega_{D, 2}^{*}\left[b_{D}-b_{L}\right]-i \exp \left(-i \Delta_{0} t\right) \Omega_{0} b_{1}^{\prime}, \\
\dot{b}_{D}=i \exp \left(-i \Delta_{1} t\right) \Omega_{D, 1} b_{1}^{\prime}+i \exp \left(-i \Delta_{2} t\right) \Omega_{D, 2} b_{2}^{\prime}, \\
\dot{b}_{L}=i \exp \left(-i \Delta_{1} t\right) \Omega_{D, 1} b_{1}^{\prime}-i \exp \left(-i \Delta_{2} t\right) \Omega_{D, 2} b_{2}^{\prime} .
\end{gathered}
$$

Thus, Eq. (10) is the same as Eq. (9) barring the change of sign in the $\Omega_{0}$ terms. Hence, by the above argument, this scenario therefore allows for chirality control when $\varepsilon_{0}(t)$ $\neq 0$.

Numerical studies indeed confirm that control over the $|D\rangle$ and $|L\rangle$ populations is, indeed, extensive when laser properties (pulse widths, central frequencies, time delay, etc.) are varied in this scenario. Consider, for example, excitation from a racemic mixture. Figure 2(a) shows the dependence of the populations of $|D\rangle$ and $|L\rangle$ on the phase $\theta \equiv \theta_{2}$ of $\varepsilon_{2}(t)$, where the pulses are assumed Gaussians [5] of the form $\varepsilon_{k}(t)=\beta_{k} \exp \left\{-\left[\left(t-t_{k}\right) / \alpha_{k}\right]^{2}\right\} \quad(k=0,1,2)$, with $\beta_{k}$ $=\left|\beta_{k}\right| \exp \left(i \theta_{k}\right)$. Clearly, one may preferentially deplete ground-state $L$ or ground-state $D$ just by changing $\theta$ by $\pi$. Similarly, Fig. 2(b) shows the extensive dependence of the $L$ and $D$ populations on the intensity ratio $\beta_{0} / \beta_{1}$.
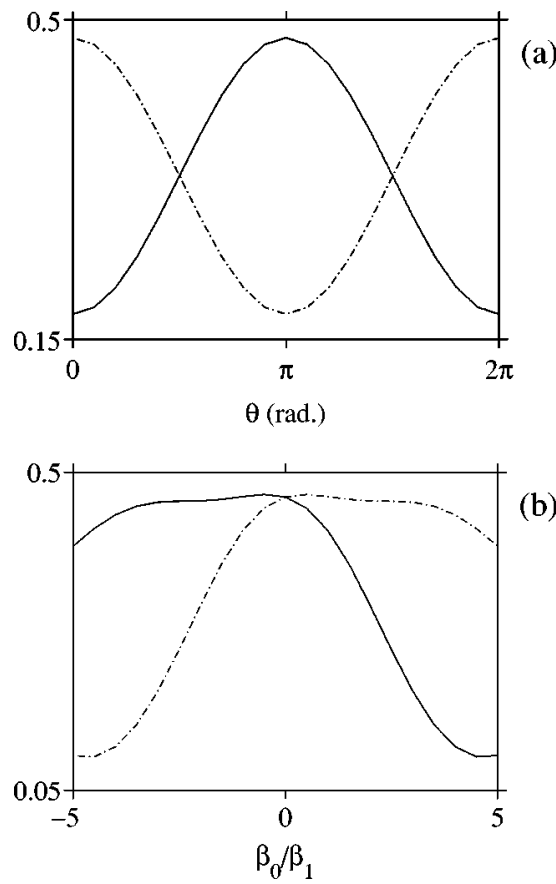

FIG. 2. Probability of observing $D$ (solid lines) and of observing $L$ (dot-dashed lines) after laser excitation of a racemic mixture as a function of (a) the phase $\theta$ of $\varepsilon_{2}(t)$ and (b) the ratio of amplitudes $\beta_{0} / \beta_{1}$. System parameters are: $\mu_{1 L}=\mu_{1 D}=1$ a.u., $\mu_{2 L}=-\mu_{2 D}$ $=1$ a.u., $\beta_{i}=1 \times 10^{-5}$ a.u., $i=1,2, \quad \alpha_{i}=2$ psec, $i=0,1,2, \Delta_{1}$ $=\Delta_{2}=-5 \mathrm{~cm}^{-1}, t_{1}=t_{2}$, and (a) $\beta_{0}=4 \times 10^{-5}$ a.u., $\Delta_{0}=0$, and $t_{0}=t_{1}$. (b) $\beta_{0}=1 \times 10^{-5}$ a.u., $\Delta_{0}=-5 \mathrm{~cm}^{-1}$, and $t_{0}-t_{1}$ $=-2$ psec.

Finally, we note that control depends upon the ability to coherently prepare excited-state levels by one or more simultaneous excitation routes, embodied in the simultaneous radiative coupling of levels $|1\rangle$ and $|2\rangle$ to one another and to $\left|D_{i}\right\rangle$ and $\left|L_{i}\right\rangle$. Thus, this constitutes a demonstration of the use of coherent control [13] to alter the overall chirality of a system without the use of chiral input. What is required experimentally is control over the phase of the electric field. One possible approach is to use ultrashort pulses [14,15] which allow defining the overall electric-field phase.

In summary, we have derived the basic principle that allows, using the dominant electric-dipole light interaction, for phase-selective transfer of a racemic mixture into the pure $D$ or $L$ enantiomeric form. In doing so, we have ignored collisional effects, as well as radiative emission to the ground state, discussed in Ref. [5], because they do not modify the principles described above.

This research was supported by the U.S. Office of Naval Research, by the Minerva Foundation, and by the German Israeli Foundation.
[1] L. D. Barron, Molecular Light Scattering and Optical Activity (Cambridge University Press, Cambridge, 1982).

[2] E.g., A. Salam and W.J. Meath, Chem. Phys. 228, 115 (1998).

[3] J. Shao and P. Hänggi, J. Chem. Phys. 107, 9935 (1997).
[4] M. Shapiro and P. Brumer, J. Chem. Phys. 95, 8658 (1991).

[5] M. Shapiro, E. Frishman, and P. Brumer, Phys. Rev. Lett. 84, 1669 (2000).

[6] The system analyzed in Ref. [5] consists of four levels coupled 
by linearly polarized light, an example being a medium composed of a single value of $M_{J}$. Note that such a system is not chiral. For a detailed discussion of the role of angular momentum in coherently controlled asymmetric synthesis, see M. Shapiro, E. Frishman, and P. Brumer (unpublished).

[7] Y. Fujimura, L. Gonalez, K. Hoki, J. Manz, and Y. Ohtsuki, Chem. Phys. Lett. 306, 1 (1999); 310, 578(E) (1999). Note, that these authors study control over an initial superposition state, rather than a racemic mixture.

[8] Y. Fujimura (unpublished).

[9] C.S. Maierle and R.A. Harris, J. Chem. Phys. 109, 3713 (1998).

[10] J.H. Shirley, Phys. Rev. 138, B979 (1965).

[11] A. Brown and W.J. Meath, J. Chem. Phys. 109, 9351 (1998).

[12] References to molecules that are proposed to have level struc- ture with these characteristics have been given in Ref. [4]. More recently, we have shown [E. Deretey, M. Shapiro, and P. Brumer (unpublished)] that 1,3 dimethylallene does display this level structure and is extensively controllable [D. Gerbasi, M. Shapiro, and P. Brumer, J. Chem. Phys. 115, 5349 (2001)] using the proposed laser scenario. Other molecules are under study.

[13] For recent reviews, see M. Shapiro and P. Brumer, Adv. At., Mol., Opt. Phys. 42, 287 (2000); S. Rice and M. Zhao, Optical Control of Molecular Dynamics (Wiley, New York, 2000).

[14] A. Apolonski, A. Poppe, G. Tempea, C. Spielmann, T. Udem, R. Holzwarth, T.W. Hänsch, and F. Krausz, Phys. Rev. Lett. 85, 740 (2000)

[15] C. Raman, T.C. Weinacht, and P.H. Bucksbaum, Phys. Rev. A 55, R3995 (1997). 